\title{
New bijective links on planar maps
}

\author{
Éric Fusy \\ Dept. Mathematics, Simon Fraser University, Vancouver. E-mail: eric. fusyeinria.fr
}

\begin{abstract}
This article describes new bijective links on planar maps, which are of incremental complexity and present original features. The first two bijections $\Phi_{1,2}$ are correspondences on oriented planar maps. They can be considered as variations on the classical edge-poset construction for bipolar orientations on graphs, suitably adapted so as to operate only on the embeddings in a simple local way. In turn, $\Phi_{1,2}$ yield two new bijections $F_{1,2}$ between families of (rooted) maps. (i) By identifying maps with specific constrained orientations, $\Phi_{2} \circ \Phi_{1}$ specialises to a bijection $F_{1}$ between 2-connected maps and irreducible triangulations; (ii) $F_{1}$ gives rise to a bijection $F_{2}$ between loopless maps and triangulations, observing that these decompose respectively into 2-connected maps and into irreducible triangulations in a parallel way.

Résumé. Cet article décrit de nouveaux liens bijectifs sur les cartes planaires. Nos constructions sont de complexité croissante et présentent des caractéristiques originales. Les deux premières bijections $\Phi_{1,2}$ portent sur des cartes orientées. Elle peuvent être vues comme des variations sur une contruction classique de posets sans $N$ à partir d'orientations bipolaires, adaptées ici pour opérer de manière très simple sur le plongement. Les bijections $\Phi_{1,2}$ entrainent à leur tour deux nouvelles bijections $F_{1,2}$ entre familles de cartes (enracinées). (i) En identifiant les cartes avec certaines orientations contraintes, $\Phi_{2} \circ \Phi_{1}$ se spécialise en une bijection $F_{1}$ entre cartes 2-connexes et triangulations irréductibles, (ii) $F_{1}$ induit une bijection $F_{2}$ entre cartes sans boucles et triangulations, qui se décomposent respectivement en cartes 2-connexes et en triangulations irréductibles de manière parallèle.
\end{abstract}

Keywords: Planar maps, bijections, orientations

\section{Introduction}

Planar maps (i.e., connected graphs equipped with a planar embedding) are a rich source of structural correspondences and enumerative results. As discovered by Tutte in the 60's (Tut63), many families of maps (eulerian, triangulations, quadrangulations...) have strikingly simple counting coefficients, essentially of binomial form (for counting purpose, all maps are assumed to be rooted, i.e., with a marked oriented edge called the root). The two main methods by now to obtain such formulas are the recursive approach introduced by Tutte (Tut73) and the bijective approach introduced by Schaeffer (Sch98). Tutte's approach has the advantage of being quite automatic, but requires an involved machinery - the quadratic methodto solve equations on generating functions. In contrast, the bijective approach yields direct combinatorial proofs (though a unifying framework is still to be found). Given a family of map $\mathcal{M}$, the idea is to exhibit a regular combinatorial structure - typically an orientation with simple outdegree conditions - specific to the maps in $\mathcal{M}$, which gives a procedure to encode each map of $\mathcal{M}$ by a spanning tree with simple degree conditions; conversely the map is recovered by performing local operations on the tree so as to 


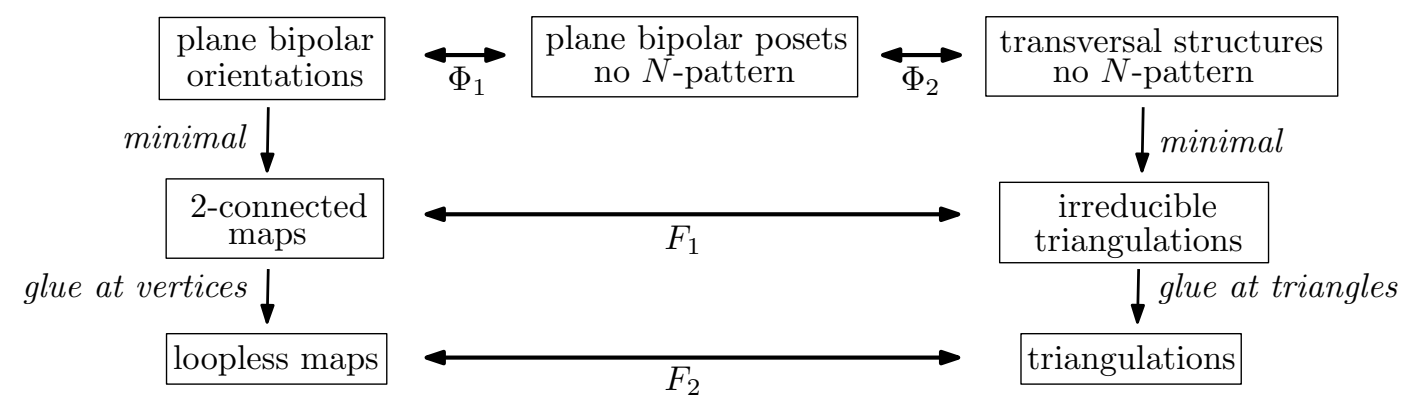

Fig. 1: Relations between the combinatorial structures considered in the article. Bijective links are indicated by double arrows

close the faces one by one. Such a bijective construction ensures that the counting coefficients of $\mathcal{M}$ are equal to those of the associated tree family, which typically have a closed formula involving binomial coefficients.

These enumerative techniques yield thus an extensive table of counting formulas for families of maps. By examining such a table, one notices that seemingly unrelated map families are equinumerous. This asks for bijective explanations as direct as possible to understand the underlying structural correspondences. On a few instances there already exist very simple bijective constructions. Such bijections are of a type different from the above mentioned bijective constructions from trees. Indeed, they are from map to map and operate directly on the embedding in a simple local way. Let us mention the classical duality construction between maps with $i$ vertices and $j$ faces and maps with $j$ vertices and $i$ faces, the radial mapping between maps with $n$ edges and 4-regular maps with $n$ vertices, and the so-called trinity mapping between bipartite 3-regular maps with $2 n$ vertices and eulerian maps with $n$ edges (the two latter constructions can be traced back to Tutte (Tut63)). We have observed two further coincidences:

(i): 2-connected maps with $n$ edges are equinumerous to so-called irreducible triangulations (triangulations of the 4-gon with no separating triangle) with $n+1$ inner vertices,

(ii): loopless maps with $n$ edges are equinumerous to triangulations with $n+3$ vertices.

In this article, we describe new bijective constructions for proving (i) and (ii). The bijection for (i) presents original features; it is nonrecursive and operates directly on the embedding in a local way, but it makes use of specific combinatorial structures and is thus less straightforward than the classical mapto-map correspondences. To wit, the bijection we describe for (i) can be seen as a direct map-to-map correspondence borrowing ideas from the above mentioned bijective constructions of maps from trees. The first step is to associate with each map-family a combinatorial structure: plane bipolar orientations for 2-connected maps and transversal structures for irreducible triangulations (these are defined in Section 27. We show in Section 3 that these structures are closely related: plane bipolar orientations correspond to specific transversal structures that are called $N$-avoiding (they avoid $N$-shaped patterns). The correspondence is a succession of two bijections $\Phi_{1,2}$, the intermediate structures being some specific plane bipolar orientations, called $N$-avoiding plane bipolar posets. The bijection $\Phi_{1}$ is interesting on its own, as it seems to be a suitable adaptation to planar maps of a classical bijective construction on partially ordered sets, see the discussion after Theorem 3.2 .

Then, the correspondence $\Phi_{2} \circ \Phi_{1}$ specialises to a bijection, described in Section 4 , between (rooted) 2- 
connected maps and irreducible triangulations. The bijection, denoted by $F_{1}$, is based on the two following properties: (a) to each 2-connected map (irreducible triangulation) is associated a canonical plane bipolar orientation (transversal structure, resp.) which is the unique one avoiding a certain pattern; such plane bipolar orientations and transversal structures are called minimal; (b) the correspondence $\Phi_{2} \circ \Phi_{1}$ matches minimal plane bipolar orientations with minimal transversal structures.

As described in Section 5, the bijection $F_{1}$ yields in turn a bijection, denoted $F_{2}$, for proving (ii). We make use of two classical decompositions that hold on rooted maps. A loopless map decomposes as a 2-connected map, called the core, and a collection of components that are smaller loopless maps; whereas a triangulation decomposes as an irreducible triangulation, called the core, and a collection of components that are smaller triangulations. The key observation is that the two decompositions are parallel, for a convenient choice of the size parameters. The bijection $F_{2}$ is thus specified recursively: the cores are matched by the bijection $F_{1}$, and the components are matched recursively by $F_{2}$. Let us mention that another bijective proof of (ii) has been described by Wormald (Wor80), still recursive but based on different principles (in (Wor80), an isomorphism is established between the generating tree of loopless maps and the generating tree of triangulations). To sum up, the whole bijective scheme of the article is shown in Figure1.

\section{Preliminaries}

\subsection{Planar maps}

A planar map, shortly called a map, is a connected unlabelled planar graph embedded in the plane with no edge-crossings, the embedding being considered up to continuous deformation. Loops and multiple edges are allowed. In addition to the vertices and edges of the graph embedded, a map has faces, which are the maximal connected areas of the plane split by the embedding. The unbounded face is called the outer face, the other ones are called inner faces. Edges and vertices are said to be inner or outer whether they are incident to the outer face or not. A map is rooted by distinguishing and orienting an edge, called the root, with the condition that the root has the outer face on its left. The origin of the root is called the root vertex. In this article we will consider the following families of (rooted) planar maps:

- Loopless maps. A loopless is a map with no loop, i.e., each edge has two distinct extremities. Multiple edges are allowed.

- 2-connected maps. A map is 2-connected if it is loopless and the deletion of any one vertex does not disconnect the map. Multiple edges are allowed.

- Triangulations. A triangulation is a map with no loop nor multiple edges and with all faces of degree 3 . These correspond to maximal planar graphs embedded in the plane.

- Irreducible triangulations. A triangulation of the 4-gon is a map with no loop nor multiple edges, with a quadrangular outer face and triangular inner faces. An irreducible triangulation of the 4-gon, shortly called irreducible triangulation, is a triangulation of the 4-gon without separating 3-cycle, i.e., the interior of any 3 -cycle is a face. 

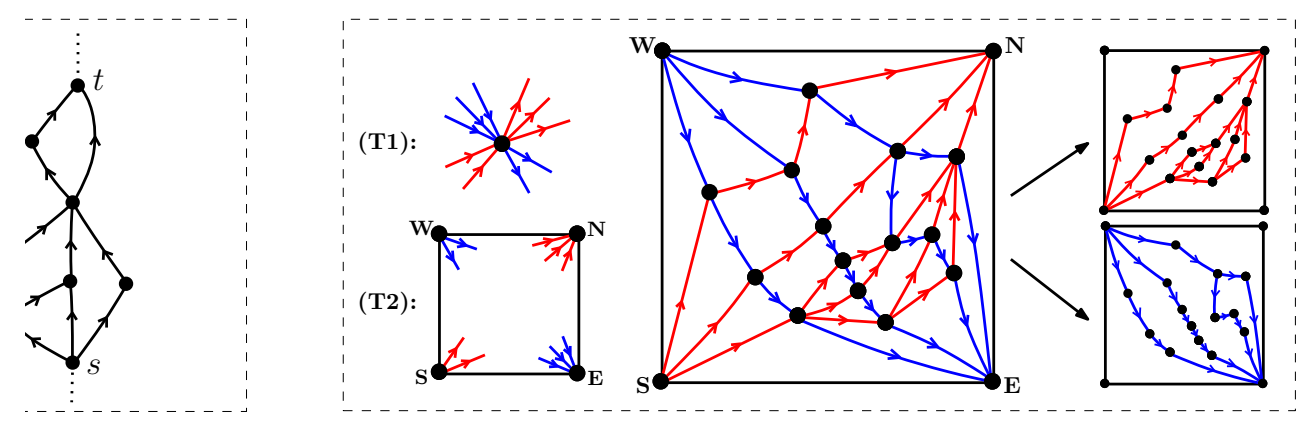

b)

Fig. 2: (a) Plane bipolar orientations: local conditions and an example. (b) Transversal structures: local conditions and an example.

\subsection{Plane bipolar orientations}

A bipolar orientation on a connected graph $G$ is an acyclic orientation with a unique source (vertex with only outgoing edges) and a unique sink (vertex with only ingoing edges). Equivalently, the partial order induced on the vertices by the orientation has a unique minimum and a unique maximum. The source and the sink, which are called the poles or the special vertices, are classically denoted $s$ and $t$. The other vertices are said to be nonspecial. It is convenient when considering plane bipolar orientations to draw two half-lines starting respectively from $s$ and $t$ and reaching into the outer face. The outer face is thus split into two unbounded faces, which are called the special faces: the one on the left (right) of the map, looking from $s$ to $t$, is the left (right, resp.) special face. Bipolar orientations constitute the natural combinatorial structure characterising 2-connectivity. Indeed, as is well known (DFOdMR95), a graph $G$ with two marked vertices $s$ and $t$ admits a bipolar orientation with source $s$ and $\operatorname{sink} t$ iff $G$ is 2-connected upon connecting $s$ and $t$ by an edge. A plane bipolar orientation is a bipolar orientation on a planar map $M$ such that the source and the sink are outer vertices of $M$. Plane bipolar orientations have the nice property that they are characterised by two simple local properties, one around vertices and one around faces, see Figure 2 (a):

(V): Around each nonspecial vertex, the edges form a nonempty interval of outgoing edges and a nonempty interval of ingoing edges.

(F): The contour of each inner face $f$ is made of two oriented paths with same origin $s_{f}$ and same end $t_{f}$. The two special faces are each bordered by a path that goes from $s$ to $t$.

Given Property (V), we can define the left (right) lateral face of a nonspecial vertex $v$ as the face incident to the angle between the last ingoing (outgoing) edge and the first outgoing (ingoing, resp.) edge in clockwise order around $v$. Given an inner face $f$, the vertices $s_{f}$ and $t_{f}$ are respectively called the source and the sink of $f$. The path from $s_{f}$ to $t_{f}$ that has the exterior of $f$ on its left (right) is called the left (right) lateral path of $f$ and is denoted $P_{\ell}(f)\left(P_{\mathrm{r}}(f)\right.$, resp.). The last edge of $P_{\ell}(f)$ is called the topleft edge of $f$ and the first edge of $P_{\mathrm{r}}(f)$ is called the bottomright edge of $f$. The vertices of $P_{\ell}(f) \backslash\left\{s_{f}, t_{f}\right\}$ $\left(P_{\mathrm{r}}(f) \backslash\left\{s_{f}, t_{f}\right\}\right)$ are called left (right, resp.) lateral vertices of $f$. The path bordering the left (right) special face is called the left (right, resp.) outer path. 
Given a plane bipolar orientation, a transitive edge is an edge whose two extremities are connected by an oriented path of length at least 2. A plane bipolar poset is a plane bipolar orientation with at least 3 vertices and with no transitive edge. The terminology refers to the fact that a plane bipolar poset is a planarly embedded Hasse diagram representing a poset.

The following property is easily checked from Condition $(\mathrm{F})$ :

Fact 1 A plane bipolar orientation with at least 3 vertices is a plane bipolar poset iff the two lateral paths of each inner face have length at least 2.

In particular, a plane bipolar poset has no multiple edges. Another remark to be used later is that each inner face $f$ of a plane bipolar poset has at least one lateral vertex on each side: the last vertex of $P_{\ell}(f) \backslash\left\{s_{f}, t_{f}\right\}$ is called the topleft lateral vertex of $f$ and the first vertex of $P_{\mathrm{r}}(f) \backslash\left\{s_{f}, t_{f}\right\}$ is called the bottomright lateral vertex of $f$. Given a plane bipolar poset, a $N$-pattern is a simple path of length 3 $\left(v_{0}, e_{1}, v_{1}, e_{2}, v_{2}, e_{3}, v_{3}\right)$, such that $e_{1}$ follows $e_{2}$ in cw order around $v_{1}, e_{3}$ follows $e_{2}$ in cw order around $v_{2}, e_{1}$ and $e_{2}$ are ingoing at $v_{1}$, and $e_{2}$ and $e_{3}$ are outgoing at $v_{2}$. A V-pattern is defined similarly, upon replacing clockwise by counterclockwise. Plane bipolar posets with no $N$-pattern are said to be $N$-avoiding; these play an important role in the bijections to be given next.

\subsection{Transversal structures}

Transversal structures play a similar part for irreducible triangulations as plane bipolar orientations for 2-connected maps.

Let us give the precise definition. Given a rooted irreducible triangulation $T$, denote by $N, E, S, W$ the outer vertices of $T$ in $\mathrm{cw}$ order around the outer face, starting from the origin of the root. A transversal structure of $T$ is an orientation and a bicoloration of the inner edges of $T$, say each inner edge is red or blue, such that the following conditions are satisfied, see Figure 2 b).

(T1): The edges incident to an inner vertex of $T$ form in cw order: a nonempty interval of outgoing red edges, a nonempty interval of outgoing blue edges, a nonempty interval of ingoing red edges, and a nonempty interval of ingoing blue edges.

(T2): The edges incident to $N, E, S$, and $W$ are respectively ingoing red, ingoing blue, outgoing red, and outgoing blue.

Transversal structures were introduced by X. He ( $(\mathrm{He} 93)$ under the name of regular edge-labellings. They were further investigated by the author (Fus05) and have many applications in graph drawing: straight-line drawing (Fus05), visibility drawing (KH97), rectangular layouts (KH97). Transversal structures characterize triangulations of the 4-gon that are irreducible. Indeed, irreducibility is necessary, and each irreducible triangulation admits a transversal structure (Fus05). Moreover, transversal structures are closely related to plane bipolar orientations by the following property:

Fact 2 Let $X$ be a transversal structure on an irreducible triangulation $T$ having at least one inner vertex. Then the oriented map $X_{\mathrm{r}}$ formed by the red edges and the vertices of $T \backslash\{W, E\}$ is a plane bipolar poset, called the red bipolar poset of $X$. Similarly, the oriented map $X_{\mathrm{b}}$ formed by the blue edges and the vertices of $T \backslash\{S, N\}$ is a plane bipolar poset, called the blue bipolar poset of $X$.

A transversal structure is called $N$-avoiding if both its red and its blue bipolar posets are $N$-avoiding. 


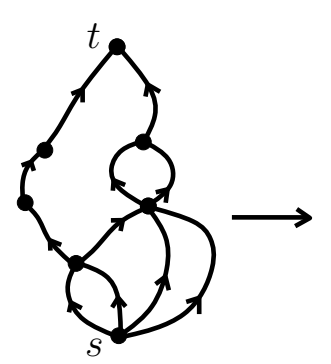

(a)

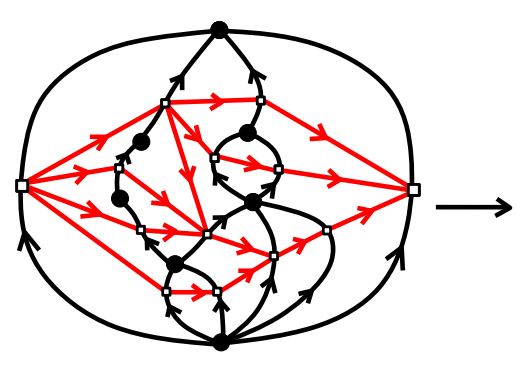

(b)

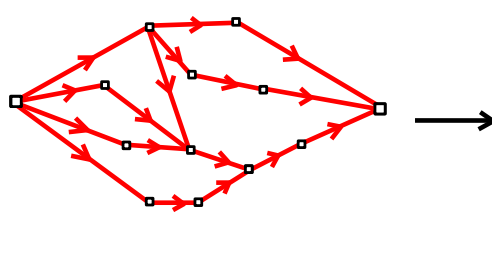

(c)

Fig. 3: (a) A plane bipolar orientation, (b) construction of (c) the associated $N$-avoiding plane bipolar poset, (d) the associated $N$-avoiding transversal structure.

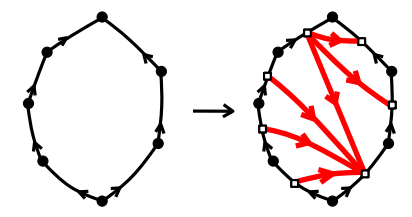

(a)

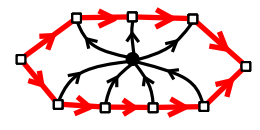

(b)

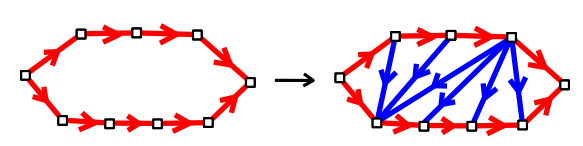

(c)

Fig. 4: (a) Insertion of the edges of $P=\Phi_{1}(O)$ inside a face of $O$. (b) Configuration of an inner face of $P$ around the associated nonspecial vertex of $O$. (c) Insertion of the blue edges of $X=\Phi_{2}(P)$ inside a face of $P$.

\section{Bijections between plane bipolar orientations and $N$-avoiding structures}

\subsection{From plane bipolar orientations to $N$-avoiding plane bipolar posets}

Let $O$ be a plane bipolar orientation. We associate with $O$ an oriented planar map $P=\Phi_{1}(O)$ as follows, see Figure 3(a)-(c).

- Add two outer edges. Add two edges $\ell$ and $r$ going from the source to the sink of $O$, so that the whole map is contained in the 2-cycle delimited on the left by $\ell$ and on the right by $r$. The augmented bipolar orientation is denoted $\widetilde{O}$.

- Insert the vertices of $P$. A vertex of $P$, depicted in white, is inserted in the middle of each edge of $\widetilde{O}$. The vertex inserted in the edge $\ell(r)$ is denoted $s_{P}\left(t_{P}\right.$, resp.).

- Insert the edges of $P$. Edges of $P$ are planarly inserted in the interior of each inner face $f$ of $\widetilde{O}$ so as to create the following adjacencies, see Figure 4 (a): the vertices of $P$ in the left lateral path of $f$ are connected to the vertex of $P$ in the bottomright edge of $f$; and the vertices of $P$ in the right lateral path of $f$ are connected to the vertex of $P$ in the topleft edge of $f$. The inserted edges are directed from the left lateral path to the right lateral path of $f$. 
Lemma 3.1 Let $O$ be a plane bipolar orientation with n edges. Then the oriented planar map $P=\Phi_{1}(O)$ is a $N$-avoiding plane bipolar poset with $n+2$ vertices. The source of $P$ is the vertex $s_{P}$ and the sink of $P$ is the vertex $t_{P}$. When $P$ and the augmented bipolar orientation $\widetilde{O}$ are superimposed, there is exactly one nonspecial vertex $v$ of $O$ in each inner face $f$ of $P$ : the outgoing edges of $v$ go to the left lateral vertices of $f$ and the ingoing edges of $v$ go to the right lateral vertices of $f$, see Figure $4 b$ ).

Conversely, given a bipolar poset $P\left(N\right.$-avoiding or not), let $O=\Psi_{1}(P)$ be the oriented planar map defined as follows:

- Insert the vertices of $O$. One vertex of $O$ is inserted in each face of $P$. The vertex inserted in the right special face is denoted $s$ and the vertex inserted in the left special face is denoted $t$.

- Insert the edges of $O$. Each nonspecial vertex $v$ of $P$ gives rise to an edge of $P$, which goes from the vertex of $O$ inside the right lateral face of $v$ to the vertex of $O$ inside the left lateral face of $v$.

The correspondence between nonspecial vertices of $O$ and inner faces of $P$, as stated in Lemma 3.1 and shown in Figure $4 \mathrm{~b}$ ), ensures that $\Psi_{1}$ is exactly the procedure to recover a plane bipolar orientation from its image $P=\Phi_{1}(O)$, i.e., $\Psi_{1}$ is the left inverse of $\Phi_{1}$. As stated next, $\Psi_{1}$ is also the right inverse of $\Phi_{1}$ :

Theorem 3.2 For $n \geq 1$ and $i \geq 0$, the mapping $\Phi_{1}$ is a bijection between plane bipolar orientations with $n$ edges and $i$ nonspecial vertices, and $N$-avoiding plane bipolar posets with $n$ nonspecial vertices and $i$ inner faces. The inverse mapping of $\Phi_{1}$ is $\Psi_{1}$.

REMARK 1. It is well known that a bipolar orientation on a graph $G$ gives rise to a partial order on the edge-set of $G$, called edge-poset, according to the precedence order of the edges along oriented paths (DFOdMR95) (observe that no embedding is needed to define this poset). It is well known in order theory that this construction is indeed a bijection between bipolar orientations on graphs and so called $\mathrm{N}$ free posets, which are posets with no induced $N$ in the Hasse diagram (HM87). Moreover, it is possible to enrich this construction so as to take a planar embedding into account: the planar embedding of the plane bipolar orientation turns to a so-called 2-realizer of the associated poset (dFdM96).

Our mapping $\Phi_{1}$ shares some resemblance with this classical bijective construction, but there are significant differences. First, our bipolar posets might have induced $N$, but only in the form of a $V$-pattern (due to the embedding, an induced $N$ can appear either as a $N$-pattern or as a $\mathrm{V}$-pattern). Second, our constructions have the nice feature that they operate directly on the embeddings. If the mapping $\Phi_{1}$ can be considered as a simple adaptation of the edge-poset construction so as to take the embedding into account, the inverse bijection $\Psi_{1}$ is truly different from the inverse $\iota$ of the edge-poset construction. Indeed, $\Psi_{1}$ operates locally on the embedding in a very simple way, whereas $\iota$ requires some considerations and manipulations on the partially ordered sets.

\subsection{From $N$-avoiding plane bipolar posets to $N$-avoiding transversal structures}

Let $\Phi_{2}$ be the mapping that associates to a plane bipolar poset $P$ ( $N$-avoiding or not) a transversal structure $X$ in the following way, see Figure 3 (c)-(d):

- Create the outer quadrangle. Insert one vertex, denoted $W$, in the left special face of $P$ and one vertex, denoted $E$, in the right special face of $P$. Connect $W$ and $E$ to the source and sink of $P$, denoted respectively by $S$ and $N$, thus creating an outer quadrangle $(W, N, E, S)$. The bipolar 
poset augmented from $P$ by insertion of the quadrangle — edges of the quadrangle oriented from $S$ toward $N$ - is denoted $\widetilde{P}$.

- Insert the blue edges. For each inner face $f$ of $\widetilde{P}$, insert (in a planar way) blue edges inside $f$ so as to create the following adjacencies, see Figure 4(c): left lateral vertices of $f$ are connected to the bottomright lateral vertex of $f$, and right lateral vertices of $f$ are connected to the topleft lateral vertex of $f$. Blue edges inside $f$ are directed from the left lateral vertices to the right lateral vertices of $f$. In other words, $f$ is triangulated by transversal blue edges in the unique way avoiding blue $N$-patterns inside $f$.

Lemma 3.3 Let $P$ be a plane bipolar poset. Then $\Phi_{2}(P)$ is a transversal structure whose red bipolar poset is $P$ and whose blue bipolar poset is $N$-avoiding.

Call $\Psi_{2}$ the mapping that associates to a transversal structure its red bipolar poset.

Proposition 3.4 For $n \geq 1$ and $i \geq 0$, the mapping $\Phi_{2}$ is a bijection between $N$-avoiding plane bipolar posets with $n$ nonspecial vertices and $i$ inner faces, and $N$-avoiding transversal structures with $n$ inner vertices and $n+i+1$ red edges. The inverse mapping of $\Phi_{2}$ is $\Psi_{2}$.

\section{Bijection between 2-connected maps and irreducible triangula- tions}

\subsection{2-connected maps as specific plane bipolar orientations}

For rooted maps, plane bipolar orientations are always assumed to have the root edge going from the source to the sink. As already mentioned in Section 2.2. bipolar orientations are naturally associated with the property of 2-connectivity; a rooted map is 2-connected iff it admits a plane bipolar orientation. Even more is true, namely, each rooted 2-connected map can be endowed with a specific plane bipolar orientation in a canonical way. Given a plane bipolar orientation, we define a left-oriented piece, shortly a LOP, as a 4-tuple $\left(v_{1}, v_{2}, f_{1}, f_{2}\right)$ made of two distinct vertices $v_{1}, v_{2}$ and two distinct faces $f_{1}, f_{2}$ of $O$ such that: $v_{1}$ is the sink of $f_{2}$ and is a left lateral vertex of $f_{1}, v_{2}$ is a right lateral vertex of $f_{2}$ and is the source of $f_{1}$ (see Figure 5 (a)).

Fact 3 ((DFOdMR95; OdM94)) A rooted map is 2-connected iff it admits a bipolar orientations. A rooted 2-connected map $M$ has a unique plane bipolar orientation with no LOP, called the minimal plane bipolar orientation of $M$.

The terminology is due to the fact that the set of plane bipolar orientations of a fixed rooted 2-connected map is a distributive lattice, the minimal element of the lattice being the bipolar orientation with no LOP, see (OdM94).

Lemma 4.1 A plane bipolar orientation O has no LOP iff $P=\Phi_{1}(O)$ has no LOP (see Figure 5 b)). 


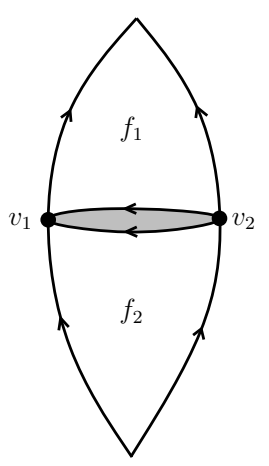

(a)

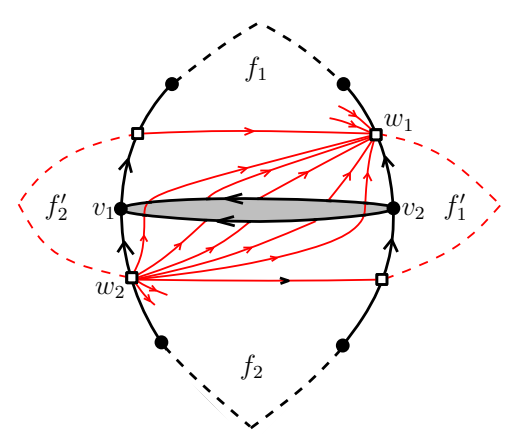

(b)

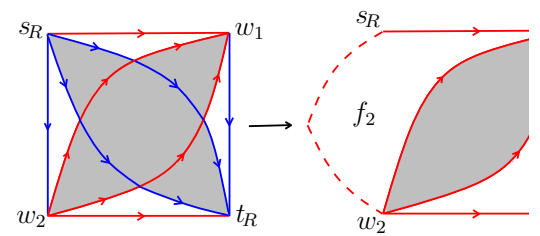

(c)

Fig. 5: (a) A left-oriented piece (LOP) of a plane bipolar orientation. (b) Each LOP in a plane bipolar orientation $O$ yields a LOP in $P=\Phi_{1}(O)$. (c) Each right alternating 4-cycle in a $N$-avoiding transversal structure $X$ yields a LOP in the red bipolar poset of $X$.

\subsection{Irreducible triangulations as specific transversal structures}

As we recall here from (Fus05), one can endow an irreducible triangulation with a specific transversal structure in a canonical way. Given a transversal structure $X$, an alternating 4-cycle of $X$ is a cycle of four edges that alternate in color, i.e., the cycle has two opposite red edges and two opposite blue edges. Condition (T1) ensures that the orientation induced on the four edges of an alternating 4-cycle $R$ has one source and one sink, which are denoted respectively by $s_{R}$ and $t_{R}$. The following properties have been proved in (Fus05) under a slightly different formulation, as only the edge-bicoloration of the transversal structure was considered:

Fact 4 Let $T$ be an irreducible triangulation endowed with a transversal structure $X$. For each alternating 4-cycle $R$ of $X$, the oriented edge-bicolored map $X_{R}$ obtained by restricting $X$ to $R$ and its interior is a tranversal structure. If $s_{R}$ is the source of outgoing red edges going inside $R$, then $R$ is called a left alternating 4-cycle. Otherwise $s_{R}$ is the source of outgoing blue edges going inside $R$, in which case $R$ is called $a$ right alternating 4-cycle.

Fact 5 A triangulation of the 4-gon is irreducible iff it admits a transversal structure. An irreducible triangulation admits a unique transversal structure with no right alternating 4-cycle, called its minimal transversal structure.

Again the terminology refers to the fact that the set of transversal structures of an irreducible triangulations is a distributive lattice whose minimal element is the transversal structure with no right alternating 4-cycle, see (Fus05). Notice that a $N$-pattern induces a right alternating 4-cycle, with no vertex inside and with the central edge of the $N$-pattern as the only edge inside. Hence any minimal transversal structure is $N$-avoiding.

Lemma 4.2 Let $X$ be a $N$-avoiding transversal structure, and let $X_{\mathrm{r}}:=\Psi_{2}(X)$ be the red bipolar poset of $X$. Then $X$ has no right alternating 4-cycle iff $X_{\mathrm{r}}$ has no LOP (see Figure $5(c)$ ). 


\subsection{The bijection}

We have gathered all the ingredients to describe the bijection between rooted 2-connected maps and rooted irreducible triangulations.

Given a rooted 2-connected map $M$ with at least 2 edges, let $T=F_{1}(M)$ be the irreducible triangulation obtained by the following steps: (i) endow $M$ with its minimal plane bipolar orientation $O$, the root edge being deleted, (ii) compute the transversal structure $X$ associated to $O$, i.e., $X:=\Phi_{2}\left(\Phi_{1}(O)\right.$ ), (iii) return $T$ as the irreducible triangulation underlying $X$, rooted at the edge going from $N$ to $E$.

According to Lemma 4.2 $X$ is the minimal transversal structure of $T$. Hence, all steps of $F_{1}$ can be inverted. Given $T$ a rooted irreducible triangulation, let $M=G_{1}(T)$ be the rooted 2-connected map obtained as follows: (i) endow $T$ with its minimal transversal structure, (ii) compute the plane bipolar orientation $O$ associated to $X: O:=\Psi_{1}\left(\Psi_{2}(X)\right)$, (iii) return the rooted 2-connected map $M$ underlying $O$ ( $M$ receives an additional root edge going from the source to the sink).

Theorem 4.3 For $n \geq 2$, the mapping $F_{1}$ is a bijection between rooted 2-connected maps with $n$ edges and irreducible triangulations with $n+3$ vertices. The inverse mapping of $F_{1}$ is $G_{1}$.

\section{Bijection between loopless maps and triangulations}

\subsection{Decomposing loopless maps into 2-connected components}

It is well known in graph theory that a connected graph $G$ decomposes in a canonical way as a collection of 2-connected components (the maximal 2-connected subgraphs of $G$ ) that are assembled by gluing components at common vertices in a tree-like fashion. This classical decomposition adapts readily to rooted loopless maps (GJ83). For our purpose it proves convenient to define the size $|M|$ of a rooted loopless map as its number of edges (counting the root) and to consider the vertex-map -made of a unique vertex and no edge - as a rooted 2-connected map; in particular, the vertex-map is loopless.

Fact 6 Each rooted loopless map $M$ is obtained in a unique way as follows.

- Take a rooted 2-connected map $C$, and order the corners of $C$ in a canonical way as $\alpha_{1}, \ldots, \alpha_{2|C|}$ (e.g., by a left-to-right d.f.s. from the root).

- Take a collection $M_{1}, \ldots, M_{2|C|}$ of rooted loopless maps. For each $1 \leq i \leq 2|C|$, insert $M_{i}$ in the corner $\alpha_{i}$ of $C$, merging the outer face of $M_{i}$ with the face incident to $\alpha_{i}$ and the root vertex of $M_{i}$ with the vertex incident to $\alpha_{i}$.

The map $C$ is called the core-map of $M$, and the $(2|C|+1)$-tuple $\mathfrak{F}(M)=\left(C ; M_{1}, \ldots, M_{2|C|}\right)$ is called the fingerprint of $M$. The size of $M$ is the sum of the sizes of the maps in the fingerprint.

\subsection{Decomposing triangulations into irreducible components}

Similarly as for loopless maps, there exists a classical decomposition of triangulations into components of higher connectivity: each triangulation on the topological sphere is obtained from a collection of 4connected triangulations (which are triangulations where all 3-cycles are facial) glued at common triangles in a tree-like fashion. The same idea - decomposition at separating 3-cycles- works as well to decompose rooted triangulations of the 4-gon into rooted irreducible components (Tut62). First let us introduce some terminology. Given a rooted triangulation $T$ of the 4-gon, denote by $N, E, S, W$ the outer 
vertices of $T$ in cw order around the outer face; then $T$ is called $W E$-diagonal if $W$ and $E$ are adjacent, is called $S N$-diagonal if $S$ and $N$ are adjacent, and is called non-diagonal otherwise. Notice that the two diagonal cases are disjoint by planarity of $T$. The unique rooted irreducible triangulation that is $W E$ diagonal ( $S N$-diagonal) is the quadrangle $(N, E, S, W)$ split by a diagonal $(W, E)((S, N))$; this map is called the $W E$-link-map ( $S N$-link-map, resp.). For our purpose it is convenient to define the size of a triangulation or of a triangulation of the 4-gon $T$ as $\|T\|:=(|V(T)|-3)$. Notice that $\|T\|$ is the number of inner vertices if $T$ is a triangulation and $2\|T\|$ is the number of inner faces if $T$ is a triangulation of the 4-gon, by Euler's relation.

Fact 7 Each rooted triangulation $T$ of the 4-gon is obtained in a unique way as follows.

- Take a rooted irreducible triangulation I, and order the inner faces of I in a canonical way as $f_{1}, \ldots, f_{2|| I||}$ (e.g., by a left-to-right d.f.s. from the root).

- Take a collection $T_{1}, \ldots, T_{2|| I||}$ of rooted triangulations. For each $1 \leq i \leq 2|| I||$, substitute $f_{i}$ by $T_{i}$ in a canonical way (e.g., the outer triangle of $T_{i}$ fits with the contour of $f_{i}$ and the root vertex of $T_{i}$ fits with a distinguished vertex of $f_{i}$ ).

The map I is called the core-triangulation of $T$; $T$ is nondiagonal if I has at least one inner vertex, is $W E$-diagonal if I is the WE-link-map, and is $S N$-diagonal if I is the $S N$-link-map. The $(2\|I\|+1)$ tuple $\mathfrak{F}(T)=\left(I ; T_{1}, \ldots, T_{2|| I \|}\right)$ is called the fingerprint of $T$. The size of $T$ is the sum of the sizes of the maps in the fingerprint.

\subsection{The bijection}

Our size-preserving bijection between rooted loopless maps and rooted triangulations is defined recursively from the bijection $F_{1}$. It is convenient to augment the correspondence $F_{1} / G_{1}$ with one object of size 1 on each side: the edge-map - made of two vertices connected by an edge- corresponds to the $W E$-link-map. We denote by $\mathcal{L}_{\leq n}\left(\mathcal{T}_{\leq n}\right)$ the family of rooted loopless maps (triangulations, resp.) of size at most $n$.

The bijection $F_{2}$ is specified recursively as follows. First, the vertex-map, the unique rooted loopless map $M$ such that $|M|=0$, is mapped by $F_{2}$ to the triangle-map, the unique rooted triangulation $T$ such that $\|T\|=0$. Given $n \geq 0$, assume that $F_{2}$ is a well defined size-preserving mapping from $\mathcal{L}_{\leq n}$ to $\mathcal{T}_{\leq n}$, i.e., $\left\|F_{2}(M)\right\|=|M|$ for every rooted loopless map $M \in \mathcal{L}_{\leq n}$. Let $M$ be a rooted loopless map of size $n+1$, with fingerprint $\mathfrak{F}(M)=\left(C ; M_{1}, \ldots, M_{2|C|}\right)$. Define $T$ as the rooted triangulation of the 4-gon whose fingerprint is $\mathfrak{F}(T)=\left(F_{1}(C) ; F_{2}\left(M_{1}\right), \ldots, F_{2}\left(M_{2|C|}\right)\right)$. By the recurrence assumption, $T$ is well defined and $\| T||=|M|$, as the sizes of $T$ and $M$ are obtained by adding up the sizes of the maps in the fingerprints. Define $\widetilde{T}:=F_{2}(M)$ as the rooted map obtained from $T$ by adding an edge from $N$ to $S$ taken as the new root. Notice that $\widetilde{T}$ is a triangulation: all faces of $\widetilde{T}$ have clearly degree 3 , and the root edge is simple (Fact 7 ensures that $S$ and $N$ are not adjacent in $T$ ). In addition, $\|\widetilde{T}\|=\|T\|$, so that $\|\widetilde{T}\|=|M|$. We have thus described recursively a size-preserving mapping $F_{2}$ from rooted loopless maps to rooted triangulations.

The inverse mapping $G_{2}$ is defined in a similar way: we simply invert the steps of $F_{2}$. First, $G_{2}$ maps the triangle-map to the vertex-map. Given $n \geq 0$, assume that $G_{2}$ is a well defined size-preserving mapping from $\mathcal{T}_{\leq n}$ to $\mathcal{L}_{\leq n}$, and assume that $G_{2}$ is the inverse mapping of $F_{2}$, i.e., $F_{2}\left(G_{2}(T)\right)=T$ for any $T \in \mathcal{T}_{\leq n}$ and $G_{2}\left(F_{2}(M)\right)=M$ for any $M \in \mathcal{L}_{\leq n}$. Let $\widetilde{T}$ be a rooted triangulation of size $n+1$, 
and let $T$ be the rooted triangulation of the 4-gon obtained by deleting the root of $\widetilde{T}$ and keeping the same root vertex. Notice that $T$ is not $S N$-diagonal. Hence, the core-triangulation $I$ of $T$ is not the $S N$-linkmap. Let $\mathfrak{F}(T)=\left(I ; T_{1}, \ldots, T_{|| I||}\right)$ be the fingerprint of $T$. The image of $T$ by $G_{2}$ is defined to be the rooted loopless map $M$ with fingerprint $\mathfrak{F}(M)=\left(G_{1}(I) ; G_{2}\left(T_{1}\right), \ldots, G_{2}\left(T_{2|| I||}\right)\right)$. By induction, $G_{2}$ is size-preserving and is the inverse mapping of $F_{2}$. To sum up, we obtain the following bijective result.

Theorem 5.1 For $n \geq 0$, the mapping $F_{2}$ is a bijection between rooted loopless maps with $n$ edges and rooted triangulations with $n$ inner vertices. The inverse mapping of $F_{2}$ is $G_{2}$.

Acknowledgements. I am very grateful to Gilles Schaeffer for his encouragements to find a new bijection between loopless maps and triangulations. I also thank Mireille Bousquet-Mélou, Nicolas Bonichon, and Nick Wormald for interesting discussions.

\section{References}

[dFdM96] H. de Fraysseix and P. Ossona de Mendez. Planarity and edge poset dimension. Europ. J. Combinatorics, 17:731-740, 1996.

[DFOdMR95] H. De Fraysseix, P. Ossona de Mendez, and P. Rosenstiehl. Bipolar orientations revisited. Discrete Appl. Math., 56(2-3):157-179, 1995.

[Fus05] É. Fusy. Transversal structures on triangulations, with application to straight-line drawing. In Proceedings of Graph Drawing'05, volume 3843 of LNCS, pages 177188. Springer, 2005. Full paper to be published in Discr. Math., available at http://arxiv.org/abs/math.co/0602163.

[GJ83] I. P. Goulden and D. M. Jackson. Combinatorial Enumeration. John Wiley, New York, 1983.

[He93] X. He. On finding the rectangular duals of planar triangulated graphs. SIAM J. Comput., 22:1218-1226, 1993.

[HM87] M. Habib and R.H. Moehring. On some complexity properties of N-free posets with bounded decomposition diameter. Disc. Math., 63:157-182, 1987.

[KH97] G. Kant and X. He. Regular edge labeling of 4-connected plane graphs and its applications in graph drawing problems. Theoretical Computer Science, 172(1-2):175-193, 1997.

[OdM94] P. Ossona de Mendez. Orientations bipolaires. PhD thesis, Ecole des Hautes Etudes en Sciences Sociales, Paris, 1994.

[Sch98] G. Schaeffer. Conjugaison d'arbres et cartes combinatoires aléatoires. PhD thesis, Université Bordeaux I, 1998.

[Tut62] W. T. Tutte. A census of planar triangulations. Canad. J. Math., 14:21-38, 1962.

[Tut63] W. T. Tutte. A census of planar maps. Canad. J. Math., 15:249-271, 1963. 
[Tut73] W.T. Tutte. The enumerative theory of planar maps. In J.N. Srivastava et al., editor, A survey of Combinatorial Theory, pages 437-448. North-Holland Publishing Company, Amsterdam, 1973.

[Wor80] N. Wormald. A correspondence for rooted planar maps. Ars Combinatoria, 9:11-28, 1980. 
